\title{
APLICAÇÃO DE ÓLEO DIESEL NA FLOTAÇÃO DE MINÉRIO DE FERRO*
}

\section{Resumo}

\author{
João Paulo Sousa Coelho ${ }^{1}$ \\ Guilherme Gonçalves dos Santos ${ }^{2}$ \\ Livia Mara Cândico ${ }^{3}$ \\ Adriana Baldessim Costa ${ }^{4}$ \\ Wellington Ribeiro Moreira ${ }^{5}$ \\ Anderson Willian Henrique Santini ${ }^{6}$
}

A flotação é uma técnica de separação baseada na afinidade das espécies minerais com as moléculas de água. Na flotação reversa de minério de ferro, utilizam-se os compostos químicos da família das aminas primárias $\left(\mathrm{R}-\mathrm{NH}_{2}\right)$, que são moléculas compostas por uma parte apolar $(\mathrm{R})$, formada por uma cadeia carbônica, e por outra polar, constituída por grupos amina $\left(\mathrm{NH}_{3}\right)$, os quais ao serem protonadas, adsorvem-se fisicamente sobre a superfície negativa das partículas de quartzo, tornando-as hidrofóbicas. O poder de "hidrofobização" das aminas está diretamente relacionado ao tamanho de sua cadeia carbônica, sendo o limitante desta grandeza a sua solubilidade em água. Dentro desse contexto e a fim de aumentar o poder "hidrofobizante" do coletor, foram realizados testes laboratoriais, utilizando soluções formadas por diferentes proporções de amina e óleo diesel para avaliar o impacto da dosagem da mistura (óleo diesel + amina) sobre o consumo de coletor e a seletividade da flotação dos finos da Mina Casa de Pedra concluindo-se que a utilização da mistura de óleo diesel + amina não prejudica o desempenho da flotação, abrindo espaço para uma redução de quase $7 \%$ do consumo de amina, insumo mais caro que o óleo diesel.

Palavras-chave: Flotação; Amina; Óleo diesel.

\section{USE OF FUEL OIL IN IRON ORE FLOTATION}

\section{Abstract}

Flotation is a separation technique based on the affinity of the mineral species with the water molecules. In reverse flotation of iron ore, use is made of chemical compounds of the family of the primary amines $\left(\mathrm{R}-\mathrm{NH}_{2}\right)$, which are molecules composed of a nonpolar (R) formed by a carbon chain, and other polar, comprising amino groups $\left(\mathrm{NH}_{2}\right)$, which upon protonated physically adsorb on the surface of the negative quartz particles, making them hydrophobic. The power of "hydrophobization" of amines is directly related to the length of the carbon chain, and the limiting of this magnitude to its solubility in water. Within this context and in order to increase the power "hydrophobizing" collector, several laboratory tests were performed using solutions composed of different ratios of amine and diesel to assess the impact of the dosage of the mixture (diesel oil amine + fuel oil) over the purity of concentrated and metallurgical recovery of the flotation of fine of Mina Casa de Pedra concluding that the use of the mixture of diesel + amine not affect the performance of the flotation, making room for a reduction of amine consumption more expensive feedstock than diesel oil.

Keywords: Flotation; Amine; Fuel oil.

1 Engenheiro Químico, Engenheiro de Desenvolvimento, Gerência de Controle Operacional de Processos (GCOP), CSN (Companhia Siderúrgica Nacional), Congonhas, Minas Gerais, Brasil.

Eng. Metalúrgico, Eng. Especialista, MBA Gestão Industrial, GCOP, CSN, Congonhas, MG, Brasil.

3 Engenheira de Produção, Engenheira de Processos, GCOP, CSN, Congonhas, MG, Brasil.

4 Engenheira de Minas, MBA em Estratégia Empresarial, Coordenadora de Processos, GCOP, CSN, Congonhas, MG, Brasil.

5 Engenheiro de Minas, Mestre em Tecnologia Mineral, Gerente de Controle Operacional de Processos, GCOP, CSN, Congonhas, MG, Brasil.

6 Administrador de Empresas, Especialista em Engenharia de Produção, Coordenador de Controle Operacional, GCOP, CSN, Congonhas, MG, Brasil. 


\section{INTRODUÇÃO}

A Flotação é um método de concentração que se baseia nos diferentes graus de afinidade das espécies minerais com a água e o ar. No caso do minério de ferro, o principal contaminante ou mineral de ganga é o quartzo e ambas as espécies são hidrofílicas, isto é, possuem afinidade com água. Portanto, para separá-los é necessária a dosagem de reagentes químicos, denominados coletores, cuja função é tornar, de forma seletiva, a superfície de um dos minerais hidrofóbicas. De acordo com Baltar [1], na flotação catiônica reversa de minério de ferro, utiliza-se como coletor as aminas primárias $\left(\mathrm{R}-\mathrm{NH}_{2}\right)$, as quais são constituídas por uma cadeia carbônica (apolar), e por outra parte polar, as aminas $\left(\mathrm{NH}_{3}\right)$, as quais ao serem protonadas, em solução aquosa, adsorvem-se fisicamente sobre a superfície negativa das partículas de quartzo, tornando-as hidrofóbicas. Ressalta-se que a carga superficial do quartzo e, por conseqüência, a seletividade do processo é governada pelo $\mathrm{pH}$ da polpa mineral [1].

De acordo com Fuerstenau [2], a redução do tamanho da cadeia lipofílica das aminas reduz a flotabilidade do quartzo, resultando num maior consumo de coletor. Já o aumento do tamanho da cadeia hidrocarbônica diminui a solubilidade dos derivados de amina em água, dificultando a sua necessária solubilização.

O óleo diesel é um derivado do petróleo constituído majoritariamente por hidrocarbonetos saturados que destilam na faixa de temperaturas de 130 a $380^{\circ} \mathrm{C}$.

A substituição parcial da amina por óleos não polares vem sendo estudada uma vez que o último é mais barato que o primeiro. Dentro desse contexto, realizaram-se testes de laboratoriais a fim de avaliar o impacto da substituição parcial da amina por óleo diesel.

Os resultados, teores de sílica do concentrado e índices de recuperação metalúrgica, foram avaliados aplicando-se a Análise de Variância (ANOVA), uma metodologia estatística que se baseia na comparação das variações das médias entre os grupos (tratamentos) com aquelas observadas dentro dos tratamentos. No presente trabalho, os tratamentos consistiram nos diferentes ensaios de flotação realizados empregando-se distintas proporções de óleo diesel e amina.

\section{MATERIAIS E MÉTODOS}

\subsection{Planejamento Experimental}

Neste trabalho, conforme apresentado na Tabela 1, avaliou-se o impacto da substituição parcial de amina (coletor) por óleo diesel sobre a pureza do concentrado, medido pelo teor de sílica do concentrado, e a recuperação metalúrgica. Para isso, foram feitos diversos ensaios de flotação utilizando-se diversas proporções de amina e óleo diesel, mantendo-se o valor do pH em 10,2 e a dosagem de amido em $1000 \mathrm{~g} / \mathrm{t}$. Os testes foram feitos em duplicata a fim de se avaliar a significância estatística da diferença entre os resultados experimentais.

Tabela 1: Planejamento Fatorial dos testes de flotação

\begin{tabular}{|c|c|c|c|c|c|}
\hline Variáveis & \multicolumn{4}{|c|}{ Proporção Amina+Óleo Diesel } \\
\hline Amina + OD & $83 \%+17 \%$ & $88 \%+12 \%$ & $93 \%+7 \%$ & $98 \%+2 \%$ & $100 \%$ \\
\hline
\end{tabular}




\subsection{Materiais e Metodologia}

Para realização dos ensaios, coletaram-se 100 litros de amostra de alimentação da planta de flotação da Usina Central da Mina Casa de Pedra. Os reagentes utilizados foram: Gritz de Milho (depressor), Amina EDA 3C, Hidróxido de Sódio para correção do $\mathrm{pH}$ e gelatinização do amido e Óleo Diesel. O volume útil da cuba da célula de flotação empregada para realização dos testes foi de $1254 \mathrm{ml}$, a rotação do impelidor foi mantida em 950 rpm e o tempo de flotação adotado, durante os testes, foi de 150 segundos.

Para gelatinização do amido empregou-se hidróxido de sódio à razão de 8 partes de amido para 1 de $\mathrm{NaOH}$. A dosagem específica de depressor praticada, em todos os testes, foi mantida em $1000 \mathrm{~g} / \mathrm{t}$. Após a gelatinização, a solução de amido foi diluída a $1 \%$.

A mistura de amina + diesel foi feita observando as proporções de óleo diesel definidas à priori no planejamento fatorial, sendo a proporção do último (óleo diesel) calculada em relação à dosagem especifica de amina de $70 \mathrm{~g} / \mathrm{t}$.

Para preparar a amostra, o primeiro passo foi homogeneizar todo o seu volume. Após a homogeneização, retirou-se uma alíquota para análise granuloquímica e do percentual de sólidos da amostra.

Em seguida, retiraram-se mais uma alíquota de $1000 \mathrm{~g}$, cujo percentual de sólido foi ajustado em $65 \%$. A alíquota foi condicionada primeiramente com amido, previamente preparado, durante 5 minutos. Já a mistura de óleo diesel + amina com composição correspondente ao respectivo ensaio, foi adicionada depois, sendo condicionada com a polpa (alíquota) por 1 minuto. Após a etapa de condicionamento, ajustaram-se o percentual de sólidos da polpa em $50 \%$ e o valor do $\mathrm{pH}$ para o respectivo valor correspondente ao ensaio.

O procedimento narrado no parágrafo anterior foi repetido durante cada ensaio.

\subsection{Caracterização da Amostra}

A Tabela 2 apresenta as características granuloquímicas da amostra utilizada durante os ensaios.

Tabela 2: Características granuloquímicas da amostra utilizada nos ensaios.

\begin{tabular}{|c|c|c|c|c|c|c|c|}
\hline Malha (mm) & Retido (\%) & Pass. Ac. (\%) & $\mathbf{F e}(\%)$ & $\mathbf{S i O}_{\mathbf{2}}(\%)$ & $\mathbf{A l}_{\mathbf{2}} \mathbf{O}_{\mathbf{3}}(\%)$ & $\mathbf{P}(\%)$ & $\mathbf{M n}(\%)$ \\
\hline $\mathbf{0 , 2 1 2}$ & 0,00 & 100,00 & 0,00 & 0,00 & 0,00 & 0,00 & 0,00 \\
\hline $\mathbf{0 , 1 5 0}$ & 0,00 & 100,00 & 0,00 & 0,00 & 0,00 & 0,00 & 0,00 \\
\hline $\mathbf{0 , 1 0 6}$ & 9,50 & 90,50 & 41,13 & 36,72 & 1,54 & 0,04 & 0,26 \\
\hline $\mathbf{0 , 0 7 5}$ & 15,50 & 75,00 & 36,90 & 44,43 & 0,60 & 0,02 & 0,08 \\
\hline $\mathbf{0 , 0 4 4}$ & 18,40 & 56,60 & 41,80 & 37,41 & 0,45 & 0,02 & 0,10 \\
\hline $\mathbf{0 , 0 4 4}$ & 56,60 & 0,00 & 55,53 & 17,57 & 0,62 & 0,02 & 0,12 \\
\hline \multicolumn{7}{|c|}{} & \multicolumn{7}{|c|}{ Global } & 48,75 & 27,20 & 0,67 & 0,02 & 0,12 \\
\hline
\end{tabular}

\section{RESULTADOS E DISCUSSÃO}

A Tabela 3 apresenta os resultados obtidos em cada ensaio e em sua respectiva réplica. Nos próximos tópicos, analisa-se através de métodos estatísticos (ANOVA), as diferenças entre os resultados experimentais. 
Tabela 3: Resultados experimentais dos ensaios de flotação

\begin{tabular}{|c|c|c|c|c|}
\hline Dosagens & RFe (\%) & SiO $_{2}(\%)$ & $\begin{array}{c}\text { RFe (\%) - } \\
\text { Rep. }\end{array}$ & $\begin{array}{c}\mathbf{S i O}_{2}(\%)- \\
\text { Rep. }\end{array}$ \\
\hline $\begin{array}{c}\text { pH 10,2 } \\
\text { Am 100\% } \\
\text { OD 0\% }\end{array}$ & 87,75 & 2,74 & 87,98 & 2,63 \\
\hline $\begin{array}{c}\text { pH 10,2 } \\
\text { Am 98\% } \\
\text { OD 2\% }\end{array}$ & 87,44 & 3,79 & 88,24 & 3,05 \\
\hline $\begin{array}{c}\text { pH 10,2 } \\
\text { Am 93\% } \\
\text { OD 7\% }\end{array}$ & 87,60 & 3,65 & 87,49 & 3,23 \\
\hline $\begin{array}{c}\text { pH 10,2 } \\
\text { Am 88\% } \\
\text { OD 12\% }\end{array}$ & 86,73 & 3,20 & 87,40 & 2,39 \\
\hline $\begin{array}{c}\text { pH 10,2 } \\
\text { Am 83\% } \\
\text { OD 17\% }\end{array}$ & 87,45 & 2,78 & 88,01 & 3,48 \\
\hline
\end{tabular}

\subsection{Análise do Teor de Sílica do Concentrado}

A Figuras 1 mostra os gráficos com a análise estatística contendo os resíduos dos resultados referentes ao teor de sílica do concentrado. Verifica-se, através do "Normal Probability Plot" que os resíduos se distribuíram normalmente. Já nos gráficos "Residual Versus Fits" e "Residual Versus Order" verifica-se que os pontos (resíduos) não apresentaram nenhum padrão aparente de comportamento. Os dois fatos citados evidenciam que não existiram "interferências externas" durante a realização dos ensaios [3].

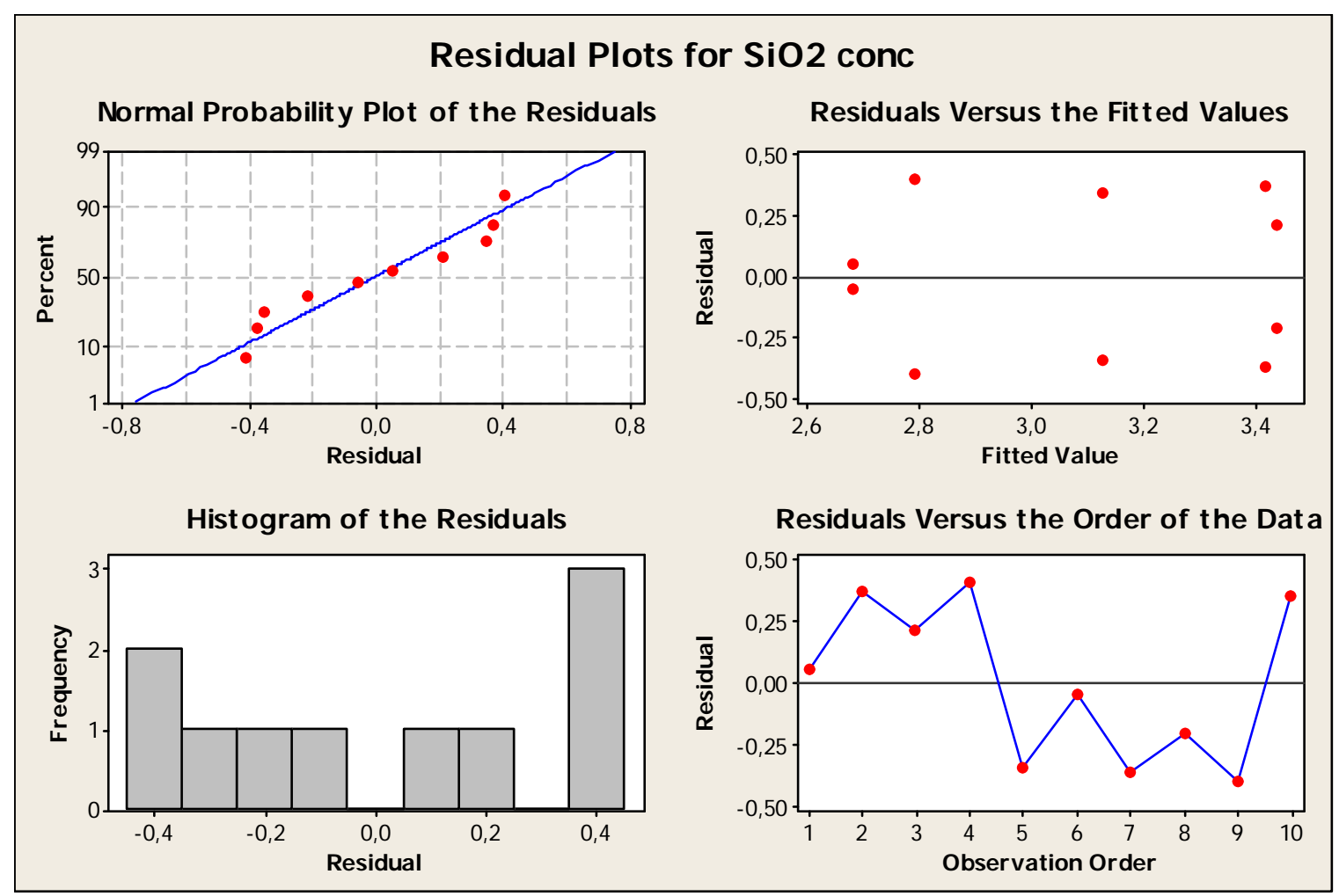

Figura 1: Gráfico com a análise estatística dos resíduos referentes ao teor de sílica do concentrado. 
Conforme revelado pela Figura 2, verifica-se através do valor de $\mathrm{P}$, maior que o nível de significância estatística adotado $(0,05)$, que a substituição parcial da amina por óleo diesel não provocou alterações relevantes sobre o teor de sílica do concentrado.

\section{One-way ANOVA: SiO2 conc versus Condição}

$\begin{array}{lrrrrr}\text { Source } & \text { DF } & \text { SS } & \text { MS } & \text { F } & \text { P } \\ \text { Condição } & 4 & 0,968 & 0,242 & 1,29 & 0,387 \\ \text { Error } & 5 & 0,941 & 0,188 & & \\ \text { Total } & 9 & 1,909 & & & \\ \text { S }=0,4338 & \text { R-Sq }=50,70 \% & \text { R-Sq (adj) }=11,27 \text { 왕 }\end{array}$

Figura 2: Análise estatística do teor de sílica do concentrado

A Figuras 3 mostra os gráficos com a análise estatística contendo os resíduos dos índices de recuperação metalúrgica. Verifica-se, através do "Normal Probability Plot" que os resíduos se distribuíram normalmente. Já nos gráficos "Residual Versus Fits" e "Residual Versus Order" é possível constatar que os pontos (resíduos) não apresentaram nenhum padrão aparente de comportamento. Os dois fatos citados evidenciam que não existiram "interferências externas" durante a realização dos ensaios [3].

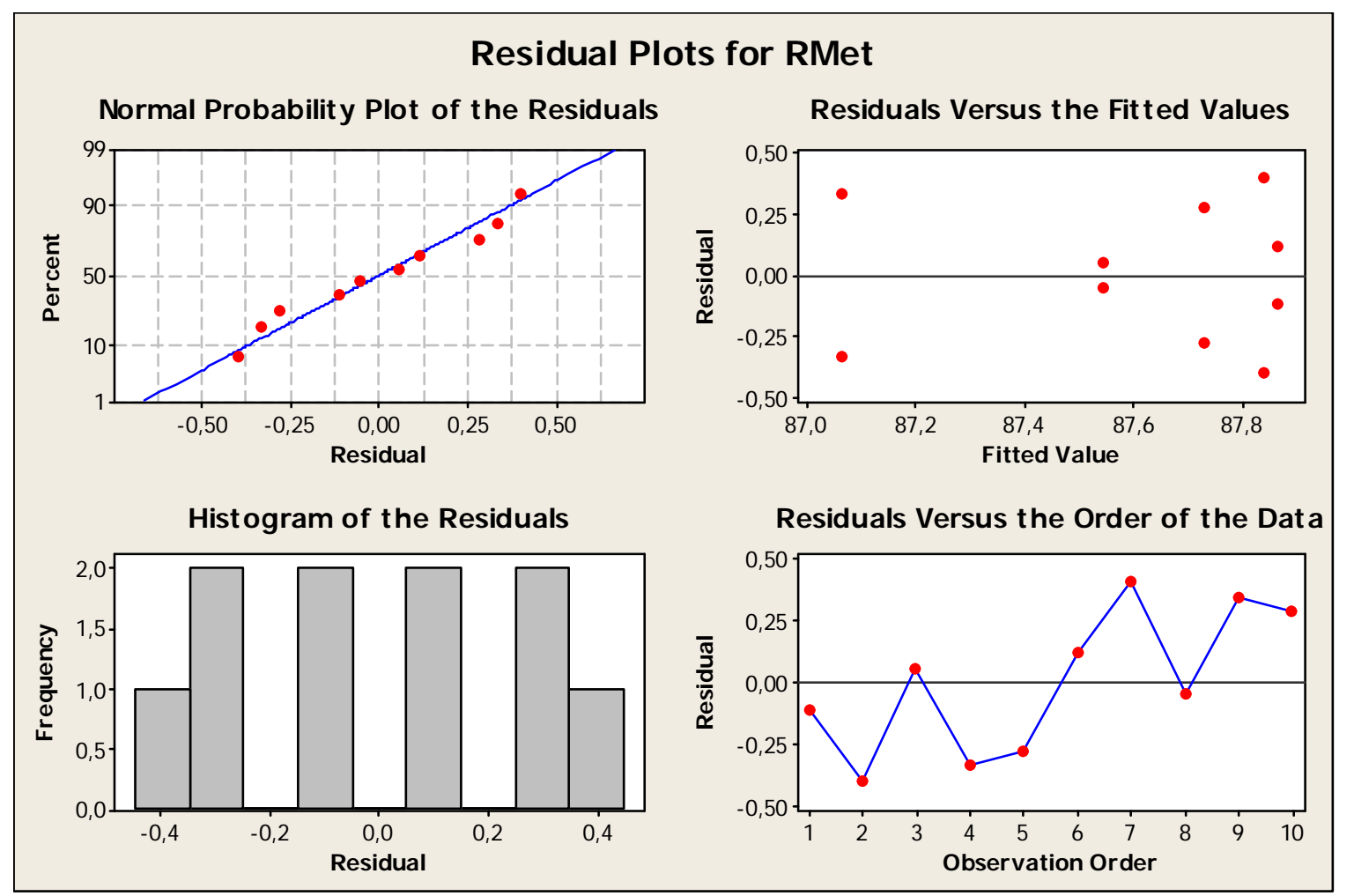

Figura 3: Gráfico com a análise estatística dos resíduos referentes aos índices de recuperação metalúrgica.

A Figura 4 apresenta os resultados da Análise de Variância (ANOVA). Conforme pode ser constatado através do valor de $P$, as diferenças observadas entre as médias dos tratamentos não foram estatisticamente significativas. Isso significa que substituição parcial de amina por óleo diesel não impactou a recuperação metalúrgica. 
One-way ANOVA: RMet versus Condição

$$
\begin{array}{lrrrrr}
\text { Source } & \text { DF } & \text { SS } & \text { MS } & \text { F } & \text { P } \\
\text { Condição } & 4 & 0,867 & 0,217 & 1,48 & 0,335 \\
\text { Error } & 5 & 0,734 & 0,147 & & \\
\text { Total } & 9 & 1,601 & & & \\
\text { S }=0,3831 & \text { R-Sq }=54,17 \text { s } & \text { R-Sq (adj) }=17,50 \text { 尔 }
\end{array}
$$

Figura 4: Análise estatística dos índices de recuperação metalúrgica

\section{CONCLUSÃO}

Conclui-se com base nos resultados experimentais que a substituição parcial da amina por óleo diesel não impactou negativamente o teor de sílica do concentrado nem os índices de recuperação metalúrgica. Dentro desse contexto e tendo em vista que o custo de aquisição do óleo diesel é significativamente inferior ao da amina, pode-se afirmar que é viável, do ponto de vista econômico, a sua substituição parcial.

É importante ressaltar que o emprego do óleo diesel na Flotação, em dosagens excessivas, pode impactar negativamente o meio ambiente, uma vez que suas moléculas não são biodegradáveis.

\section{REFERÊNCIAS}

1 BALTAR, C.A.M. Flotação no tratamento de minérios. 2. ed. Recife: Universitária da UFPE, 2008.

2 FUERSTENAU,D.W. Zeta potentials in the flotation of oxide and silicate minerals.Advances in Colloid and Interface Science, California, p.114-115, Marc. 2005. MONTGOMERY, Douglas C.Applied. Statistics and probability for engineers / Douglas C. Montgomery, George C.Runger. 3th ed. Arizona: John Wiley \& Sons, Inc, 2008. 